\title{
Role of Additional Coronal Magnetic Resonance Imaging in Decompression and Reconstruction with or without Segment Salvage in Thoracic Spine Tuberculosis
}

\author{
Sudhir Srivastava, Nandan Marathe, Sunil Bhosale, Prajakta Bhide, \\ Shaligram Purohit, Chetan Shende, Balgovind Raja \\ Department of Orthopedics, Seth GS Medical College and KEM Hospital, Mumbai, India
}

\begin{abstract}
Study Design: Retrospective and observational study.
Purpose: The present study aimed to develop guidelines for segment salvage or sacrifice based on the amount and status of the remnant portion of the vertebra as assessed by coronal magnetic resonance imaging (MRI) in addition to sagittal and axial images in thoracic spine tuberculosis (TB).

Overview of Literature: Indications for surgery have not changed significantly since the 'middle path regimen' was proposed by Tuli. Goals of modern surgical management of spinal TB include debridement of diseased vertebrae, spinal cord decompression, deformity correction, and spine stabilization. However, the extent of decompression has not been defined previously. Too less decompression will lead to compromised neurological recovery, whereas large extent of decompression is associated with increased surgical morbidity and longer segment to reconstruct.

Methods: Sixty-five patients with thoracic spine TB were divided into two groups (segment salvage/sacrifice) based on the thickness of the subchondral bone and endplate morphology of the vertebra as seen on MRI. The operative procedure in the form of instrumentation with Hartshill loop rectangle and sublaminar wire using the simultaneous anterior posterior approach in lateral position (versatile approach) was performed. The patients were analyzed for postoperative fusion, improvement in kyphosis angle, and followed up for development of complications.

Results: This method of deciding the level of fixation and segment salvage based on coronal MRI in addition to the sagittal and axial images provided good result in 64 of 65 patients, except for one patient in the segment sacrifice group who had graft buckling and resultant kyphosis.

Conclusions: For segment salvage, having a clear three-dimensional idea about the viable remnant bone is important. Viable salvaged segment reduces the morbidity of the procedure, length of the construct, and unnecessary debridement without compromising on the neural recovery and fusion rate. Hence, additional cone down coronal cuts must be required when MRI is suggestive for spinal TB because it will help in the decision making.
\end{abstract}

Keywords: Coronal magnetic resonance imaging; Segment salvage; Thoracic; Tuberculosis

Received Jan 13, 2019; Revised Mar 16, 2019; Accepted Mar 18, 2019

Corresponding author: Nandan Marathe

Department of Orthopedics, Seth G.S. Medical College and K.E.M. Hospital, Parel, Mumbai-12, Maharashtra, India

Tel: +91-7738455733, Fax: +91-022-24107463, E-mail: nandanmarathe88@gmail.com 


\section{Introduction}

Spinal tuberculosis (TB) is known since ancient times and has been documented even in 5,000-year-old Egyptian mummies. The first modern case of spinal TB was described in 1779 by Percival Pott; hence, spinal TB is also known as Pott spine [1]. The spine is the most common site for the development of skeletal TB and accounts for $50 \%$ of cases of skeletal TB. What makes spinal TB particularly alarming is the risk of neurological complications, varying from $10 \%$ to $43 \%$ [1]. Any part of the spine can be affected [2-4]; however, the thoracic spine and thoracolumbar junction are the most commonly affected areas. Thoracic spine TB is associated with neurological deficits due to the narrower spinal canal and physiological kyphosis, forcing necrotic tissue into the spinal canal.

Management strategies in spinal TB are undergoing a paradigm shift with the advent of multidrug-resistant (MDR) TB, frequency of infection in immunodeficient individuals, more accurate imaging modalities, and advances in spinal reconstruction techniques [5]. Advanced imaging techniques, such as magnetic resonance imaging (MRI), make early diagnosis of spinal TB easier, and a considerable number of patients with spinal TB are diagnosed earlier and treated more effectively before significant neurological deficits develop.

The radiological picture may not always correlate with the clinical severity of the disease in case of spinal TB. MRI may show enhanced intensity changes. There are no guidelines or set protocols regarding the extent of decompression on the basis of the radiological picture. Orthopedic surgeons are in a dilemma because too less decompression may be associated with compromised neurological recovery, whereas too much extent of decompression is associated with surgical morbidity and a longer segment to reconstruct. How to maintain the right balance is the problem. Surgeons usually follow an individualistic approach based on the pattern of bone destruction and the component of neural compression.

The present study aimed to develop guidelines for segment salvage or sacrifice based on coronal MRI imaging in addition to sagittal and axial images.

\section{Materials and Methods}

This retrospective study was conducted at Seth G. S. Medical College and K.E.M. Hospital, Mumbai, India with a study duration of 14 years. Between January 2003 and December 2016, 65 consecutive patients with thoracic spine TB underwent surgery. A single surgeon operated on all the patients. The ethics committee of Department of Orthopedics, Seth G. S. Medical college and K. E. M. Hospital, Mumbai approved this study (2015/05/30-DRB), and all the participants provided written informed consent for the surgical procedure. Indications for surgery were presence of neurologic deficit (60 patients) and unstable vertebral column (five patients). We defined an unstable vertebral column as complete loss of $>1$ vertebral body with localized kyphosis of $>30^{\circ}$, with or without coronal/ sagittal plane translation. This study included 38 male and 27 female patients, with a mean age of 27 years (range, 6-58 years).

All the patients with Frankel grade C or D were put on bed rest, nutritional supplements, chest physiotherapy, and underwent chemotherapy for a minimum period of 3 weeks before being considered for surgery as per the institutional protocol. This minimum period of 3 weeks is considered the 'middle path regime' popularized by Tuli [6]. Surgery is considered if patients fail to improve after 3-4-weeks anti-tuberculosis therapy (ATT) and bed rest [7]. Only patients who did not recover or who deteriorated while on conservative treatment were considered for surgery. Patients with Frankel grade A or B underwent surgery as early as possible.

\section{Radiological evaluation}

Preoperative radiographs included standing anteroposterior (AP) and lateral views of the thoraco-lumbar spine. Supine radiographs were acquired in patients with no ambulatory power in their lower limbs and imminent paralysis. Preoperative MRI was performed for all the patients. The patients were divided into two groups based on MRI imaging (Fig. 1). All the patients were investigated using $\mathrm{X}$-ray and MRI taken 1 week preoperatively. Surgical decision making was based on the new radiographic images.

\section{1) Group $A$}

Group A is to be considered for segment salvage (Fig. 2): (1) minimum 5-mm thickness of remnant subchondral bone; (2) absence of pus cavity within critical zone of 5 mm; (3) absence of pathological fracture/dissolution/ breach of subchondral bone in any of its area with exposed disc; and (4) no involvement of adjacent disc. 


\section{2) Group B}

Group B is to be considered for segment sacrifice: (1) remnant subchondral bone $<5 \mathrm{~mm}$ and invariably breached by abscess or granulation tissue (Fig. 3); (2) exposed disc at another location with wafer-thin subchondral bone (Fig. 4); and (3) pathological fracture of subchondral bone with necrotic adjacent disc (Fig. 5).
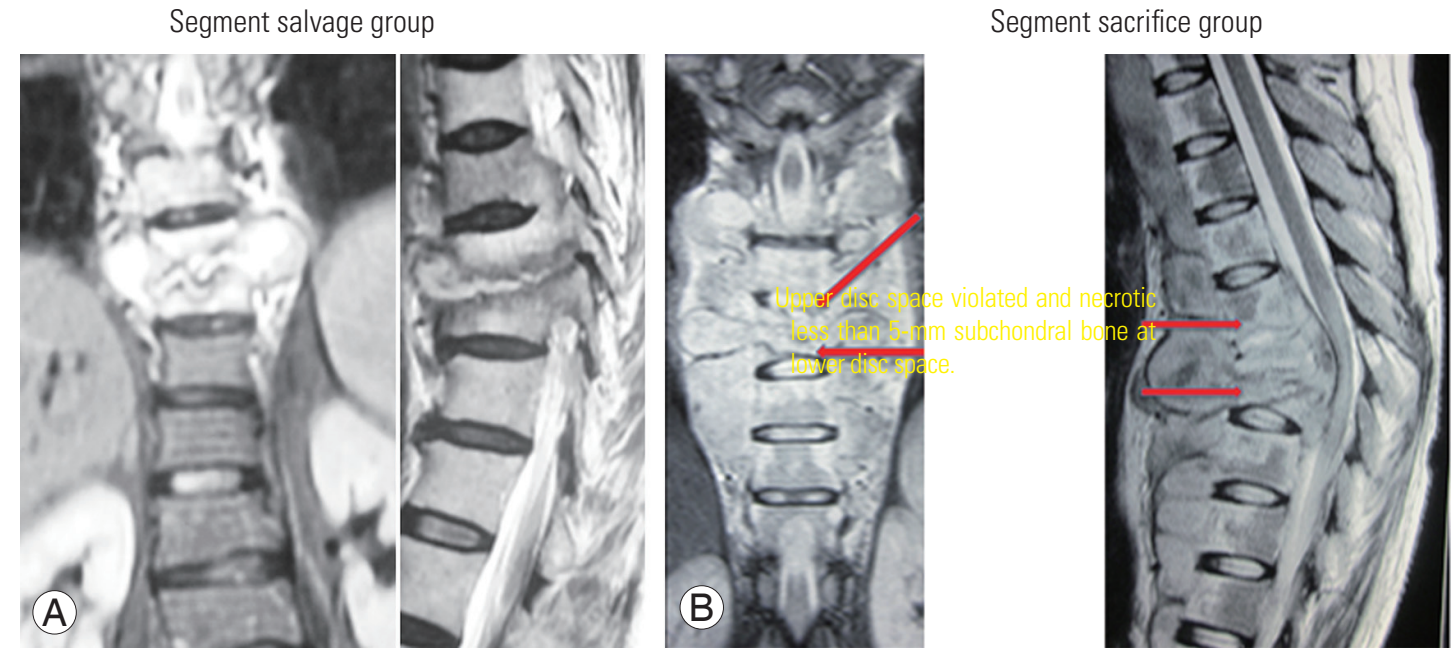

Fig. 1. (A, B) Magnetic resonance imaging picture of the groups to be considered for segment salvage and sacrifice.
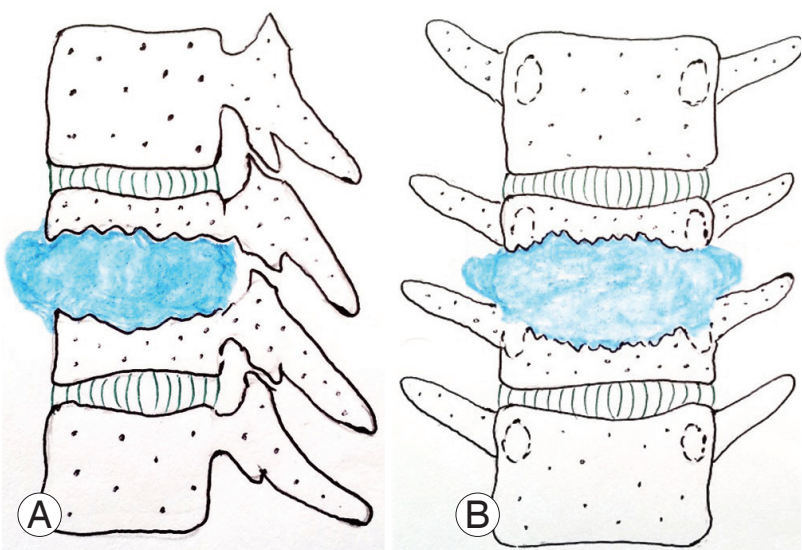

Fig. 2. (A, B) Group to be considered for segment salvage.
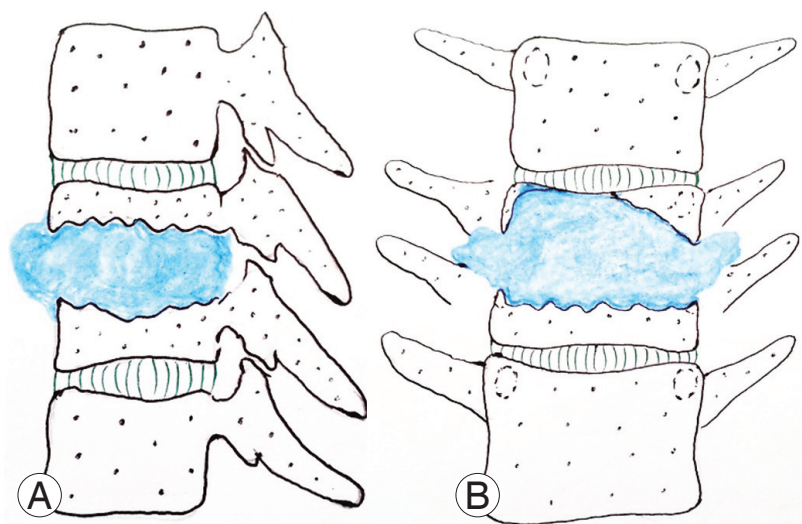

Fig. 3. (A, B) Remnant subchondral bone breached by abscess.
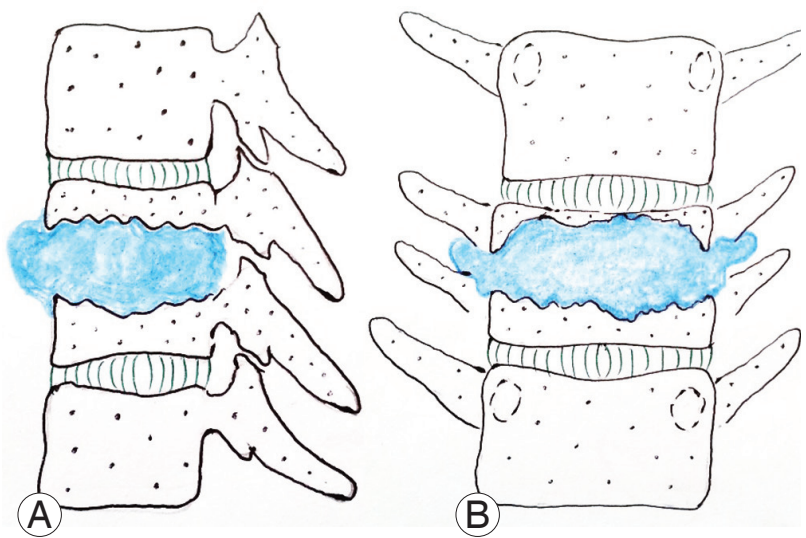

Fig. 4. (A, B) Exposed disc with wafer-thin subchondral bone.
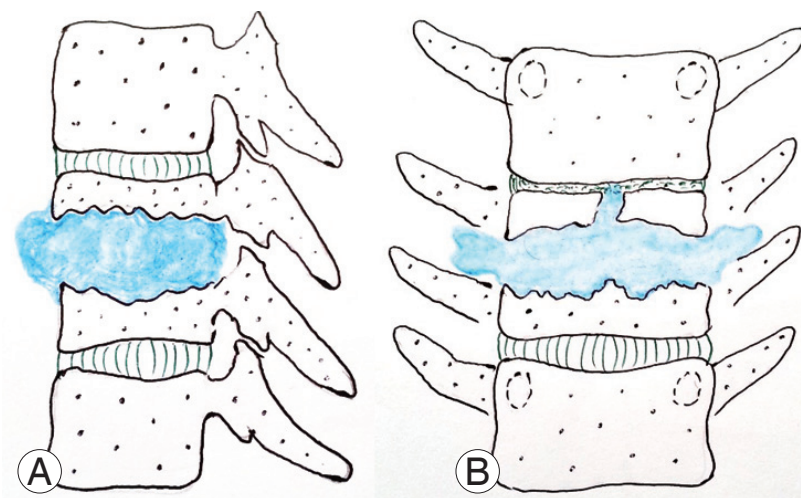

Fig. 5. (A, B) Pathological fracture of subchondral bone with necrotic adjacent disc. 


\section{Surgical procedure}

Simultaneous anterior and posterior approach in lateral position, the Versatile approach [8], was used to expose the lesion in all the patients.

\section{1) Positioning of the patient}

The patient was positioned on their left side, with their right arm placed as forward as possible on a side support. This is important for pulling the scapula anteriorly and superiorly.

\section{2) Incision and anterior exposure}

A semilunar incision was made in the right para-median region. The incision ends were slanted gradually and run close to the midline, extending along the entire length of the proposed area of fixation. The skin, subcutaneous tissue, deep fascia, and trapezius were cut along the skin incision. The rhomboids were incised toward the midline. The scapula and attached muscle were retracted anteriorly, exposing the outer surface of the rib cage. The rib corresponding to the collapsed vertebral level was excised. At this stage, an extra-pleural or trans-pleural approach was used to reach the anterior surface of the vertebral body. A chest spreader was used to increase the space; the lung was retracted on a mop with a Deaver retractor. Segmental vessels were exposed and ligated.

3) Decompression, posterior exposure, and instrumentation Debridement and de-bulking of the anterior necrotic tissue were performed. Posterior exposure of the spinous process, laminae, transverse processes, and facet joints was performed by separating the erector spinae muscle on the left and right sides. At this stage, posterior instrumentation was used. Hartshill loop rectangle and sublaminar wires were used for instrumentation in all the patients. Localized kyphosis if present was corrected.

4) Reconstruction (intra-operative decision making for segment salvage/sacrifice)

The quality of the remnant bone was assessed. If the remnant bone was $>5 \mathrm{~mm}$ and has not been breached by the tuberculous abscess, it was salvaged, and a measured length strut graft was put and impacted after neural decompression. If the remnant bone was $<5 \mathrm{~mm}$ or breached by the tuberculous abscess, it was excised, along with the adjoining disk, until the adjacent vertebral endplate was seen. A cut-off of $5 \mathrm{~mm}$ was chosen because the chances of a nonunion are increased if the remnant bone is $<5 \mathrm{~mm}$ or has been breached by necrotic tissue; in such a scenario, it is better to excise such remnant flake of bone and perform the reconstruction using healthy bone. A proper slot was made in the proximal and distal regions of the healthy bone; strut grafting was performed. Small corticocancellous chips were packed anterior to the strut graft. An additional onlay graft (part of the excised rib) was placed between the vertebral body and the anterior longitudinal ligament to span the diseased area. Subsequently, posterior bony reconstruction (fusion) was performed.

\section{5) Closure}

A chest tube was inserted, and the chest was closed. Another drainage tube was inserted into the posteriorly exposed area (near the posterior midline).

\section{6) Postoperative management}

The patient was advised to change positions while in bed to prevent pressure necrosis of the wound. The patient was mobilized after drain removal using a thoraco-lumbosacral orthosis (TLSO). Standing and walking were started once the patient regained ambulatory power. Deep vein thrombosis (DVT) prophylaxis was not administered to any patient. We had a team approach wherein immobile patients were treated by passive range of motion exercises, centripetal massage, and DVT stockings by the physiotherapists and by teaching the patients' relatives.

\section{Case record form}

A case record form was made wherein the patient's name, age, sex, vertebral level of involvement, and group (segment salvage/sacrifice) was noted. Pre- and postoperative kyphosis angles ( $\mathrm{K}$ angle), pre- and postoperative neurological status (Frankel grading), and postoperative complications if any were noted during follow-up.

AP and lateral radiographs of the thoraco-lumbar spine were performed postoperatively and during follow-up at 3, 6, 12, and 24 months. The $\mathrm{K}$ angle was calculated by drawing lines from the upper to the lower border of the first normal vertebra above and below the diseased area, respectively. The $\mathrm{K}$ angle does not represent overall thoracic kyphosis; however, it is a measure of the localized kyphosis due to the anterior column collapse from TB. Computed tomography (CT) was performed 1 year 
postoperatively in patients in whom fusion could not be assessed on X-ray. Anterior fusion was assessed by the presence of the bone bridging the vertebrae immediately proximal and distal to the reconstructed area.

\section{Statistical analysis}

SPSS software ver. 17.0 (SPSS Inc., Chicago, IL, USA) was used to calculate descriptive statistics, including the means and standard deviations, were calculated for variables. Paired $t$-test was used to perform comparison between pre- and postoperative $\mathrm{K}$ angles.

\section{Results}

All the 65 patients underwent a minimum follow-up of 18 months, with a mean follow-up of $50.23 \pm 24.46$ months (range, 18-153 months). During presentation, the mean value of preoperative kyphosis was $22.02^{\circ} \pm 8.49^{\circ}$ (range, $\left.8^{\circ}-49^{\circ}\right)$. The mean number of vertebral bodies involved was $3.16 \pm 0.37$ (range, 2-4), with D5-6 being the most commonly involved level (Table 1).

Of the 65 patients, the segment was salvaged and sacrificed in 40 and 25 patients, respectively. Anterior reconstruction was performed by using only rib grafts in 25

Table 1. Mean and range of the patient data

\begin{tabular}{lc}
$\begin{array}{l}\text { Variable } \\
\text { Follow-up duration (mo) }\end{array}$ & $\begin{array}{c}\text { Mean } \pm \text { standard deviation } \\
\text { (range) }\end{array}$ \\
\hline Preoperative kyphosis $\left(^{\circ}\right)$ & $50.23 \pm 24.46(18-153)$ \\
Vertebral bodies involved & $22.02 \pm 8.49(8-49)$ \\
Total levels fixed & $3.16 \pm 0.37(2-4)$ \\
\hline
\end{tabular}

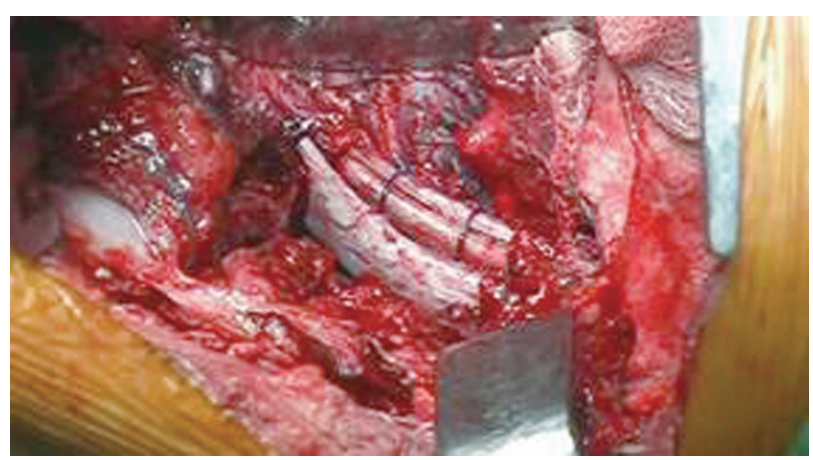

Fig. 6. Two or three measured struts of ribs tied together trans-osseously to reconstruct the full width of the body. patients (where 2 or 3 measured struts of ribs were tied together trans-osseously to reconstruct the full width of the body) (Fig. 6), tricortical iliac crest bone grafts plus ribs in 38 patients, and fibulas plus ribs in two pediatric patients. No laminectomy was required for 48 patients. Laminectomy was indicated in patients with posterior compression only. Single- and two-level laminectomies were required for 15 and two patients, respectively. The total levels fixed were $5.90 \pm 1.01$ (range, 4-9). The mean immediate postoperative $\mathrm{K}$ angle was $9.15^{\circ} \pm 4.16^{\circ}$ (range, $\left.1^{\circ}-23^{\circ}\right)$. Improvements in $\mathrm{K}$ angle were statistically significant $(p<0.001)$.

Five patients did not have any preoperative neurological deficits. Their neurological status was unchanged postoperatively. All the five patients with Frankel grade D recovered completely. Of 32 patients with Frankel grade C, 25 and seven patients recovered fully and to grade $\mathrm{D}$, respectively. Of 28 patients with Frankel grades A and B, 20 patients recovered completely, seven patients recovered to grade $\mathrm{D}$, and one patient remained paraplegic. No patient deteriorated postoperatively (Table 2).

In all cases, fusion was achieved. Two patients in group B (segment sacrifice) had superficial wound infection; one healed by daily cleaning and dressing, whereas the other required secondary suturing. One patient developed buckling of the anterior rib graft, with reappearance of kyphosis (Fig. 7). However, revision surgery was not opted for because this patient recovered neurologically from Frankel grade D to grade A and had a negligible cosmetic deformity. The patient was mobilized with a TLSO until union was achieved. We did not have any case of symptomatic DVT in our series.

All the patients underwent biopsy preoperatively, and the sample was investigated by TB MGIT (mycobacteria growth indicator tube), and drug sensitivity was determined. We had six cases of MDR TB in our series who were administered AKT as per the drug sensitivity for a longer duration (2 years) compared with 18 months for

Table 2. Pre- and postoperative neurological status of patients

\begin{tabular}{lcl} 
No. of patients & $\begin{array}{c}\text { Preoperative } \\
\text { Frankel grade }\end{array}$ & $\begin{array}{l}\text { Postoperative } \\
\text { Frankel grade }\end{array}$ \\
$n=5$ & D & E \\
$n=32$ & $C$ & $E(25), D(7)$ \\
$n=28$ & A, B & $E(20), D(7), A(1)$ \\
\hline
\end{tabular}



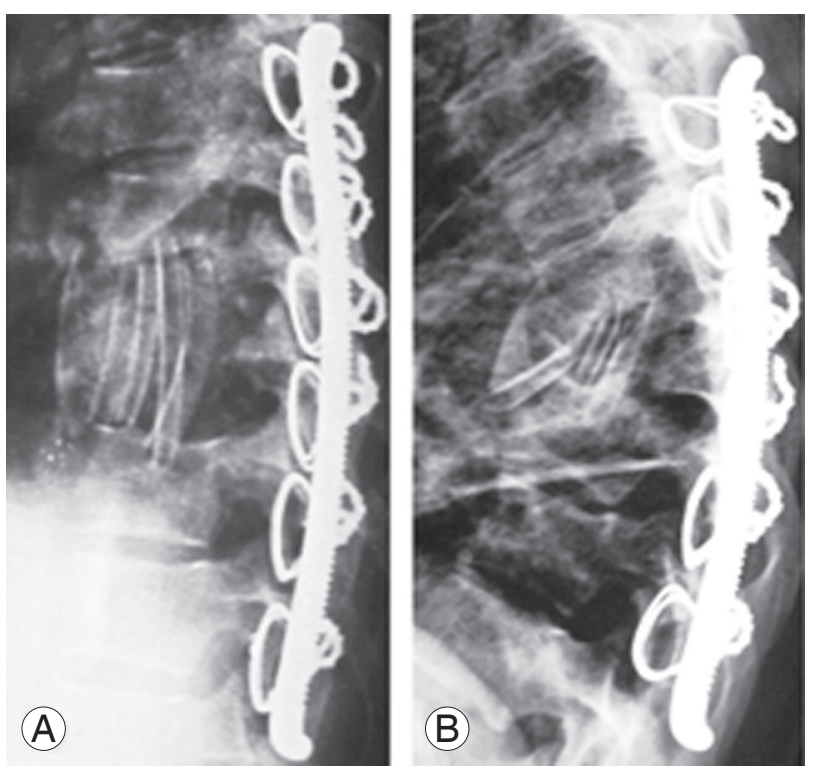

Fig. 7. (A) Immediate postoperative $X$-ray showing anterior reconstruction with multiple rib grafts. (B) Buckling of graft with localized kyphosis.

non-MDR cases. Hence, the extent of surgical debridement did not vary based on the MDR status because TB is essentially a medical disease, and appropriate AKT as per the drug sensitivity for an adequate duration forms the mainstay of treatment.

\section{Discussion}

Spinal TB is a medical disease, and ATT has a main role in the recovery and response of patients [9-16]. Only antiTB chemotherapy has been shown to be effective even in the presence of a paravertebral abscess in many patients in the absence of neurologic deficit, instability, and deformity [16-19]. General guidelines recommend confirming the diagnosis by biopsy and to start anti-TB chemotherapy based on the drug sensitivity report. Early pharmacological treatment for an adequate duration can prevent severe complications of spinal TB [9]. Combination of rifampicin, isoniazid, ethambutol, and pyrazinamide for 2 months, followed by combination of rifampicin and isoniazid for 18 months is the most frequent protocol used for treatment of spinal TB [10]. Short-course chemotherapy regimens have been demonstrated to have excellent results, except in patients younger than 15 years with an initial angle of kyphosis of $>30^{\circ}$ and whose kyphosis increases substantially [20].

The indications for surgery have not changed signifi-
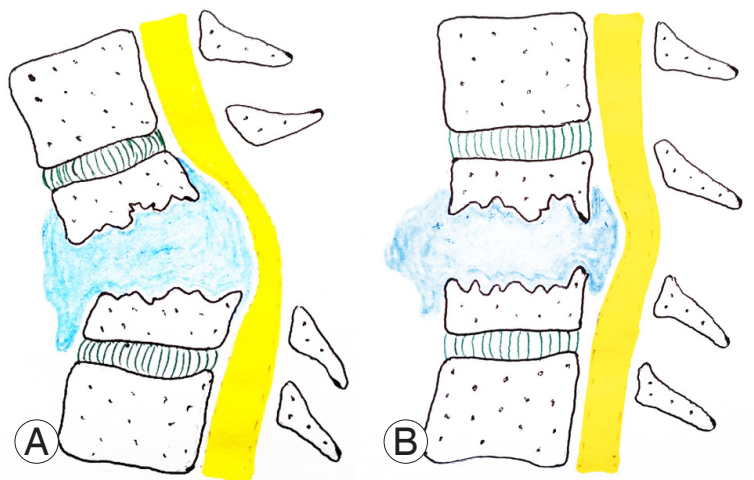

Fig. 8. (A, B) Radiologically visible compression because the tilt disappears once the deformity is corrected.

cantly since the 'middle path regimen' was proposed by Tuli [6] in 1975 for spinal TB. This included four main criteria: (1) no sign of progressive neurological recovery despite a fair trial of conservative therapy for 3-4 weeks; (2) development of neurological complications during therapy; (3) patients with pre-vertebral cervical abscess, difficulty in deglutition, and respiration; and (4) advanced neurological involvement, with flaccid paralysis, severe flexor spasm, and bowel-bladder involvement. At our institute, we also operated on pediatric patients with two or more 'spine at risk' signs and in cases with unabated destruction in junctional areas.

The goals of modern surgical management of spinal TB include debridement of diseased vertebrae, spinal cord decompression, deformity correction, spine stabilization, and further spinal cord protection. A radiological appearance of compression by an angulated viable remnant segment should not be excised on the first surgery. Once instrumentation is done, the tilt is corrected, resulting in no compression (Fig. 8). Hence, this viable remnant segment should be salvaged.

However, the extent of decompression has not been defined previously. Too less decompression will lead to compromised neurological recovery, whereas large extent of decompression is associated with increased surgical morbidity and longer segment to reconstruct. The present study aimed to define indications for segment salvage or sacrifice based on endplate morphology as defined on coronal MRI in addition to sagittal and axial images.

MRI is the neuroimaging of choice for spinal TB. MRI is more sensitive than X-ray and more specific than CT in the diagnosis of spinal TB. MRI readily demonstrates involvement of the vertebral bodies, disc destruction, cold 
abscess, vertebral collapse, and spinal deformities. MRI is also useful in detecting intramedullary or extra-medullary tuberculoma, spinal cord cavitation, spinal cord edema, and possibly unsuspected noncontiguous lesions of the spine.

Here, we used coronal MRI in addition to the sagittal and axial images to define the morphology of the endplate of the vertebra and provide a three-dimensional idea regarding the status of the remnant subchondral bone. Patients with a minimum of 5-mm thickness of remnant subchondral bone, absence of pus cavity within critical zone of $5 \mathrm{~mm}$, absence of pathological fracture/dissolution/breach of subchondral bone in any of its area with exposed disc and no involvement of adjacent disc were considered for segment salvage, and shorter construct was used. In patients with remnant subchondral bone $<5 \mathrm{~mm}$ and invariably breached by abscess or granulation tissue, exposed disc at another location with wafer-thin subchondral bone and pathological fracture of subchondral bone with necrotic adjacent disc segment was sacrificed, and a longer construct was used.

The approach described in this study is a single incision 'versatile approach' [8]. Using a single incision and the same patient position, this method offers the advantages of anterior and posterior approaches. We have used Hartshill loop rectangle with sublaminar wires in all the patients who underwent surgery in this series. Hartshill loop rectangle provides segmental fixation and corrects the deformity by sequential tightening of the sublaminar wire on a contoured Hartshill rectangle. In significantly inflamed and osteoporotic bone in TB, this method of fixation provides excellent stability being a surface fixation. The disadvantage of this approach is pedicular screw fixation is not recommended because the patient is placed in the lateral position. However, because we experienced one implant failure only, fixation with Hartshill loop rectangle and sublaminar wires is adequate if performed properly. Fixation with a Hartshill loop rectangle has the advantage of lower cost than with pedicular screws; hence, it is a suitable option, particularly in developing countries. We would like to emphasize that sound anterior reconstruction is the key to successful surgical management of spinal TB. The 'versatile approach' offers the advantage of direct visualization of the anterior reconstruction.

A slot is created in the proximal and distal bone before putting the graft, ensuring that the graft snugly fits into the anterior column and providing a wide surface area for a successful union. Anterior reconstruction may be performed via a rib graft only if the void is small, and the rib is not very osteoporotic. A tricortical graft may be harvested from the iliac crest and used for anterior reconstruction if the anterior void is large, and the rib quality is poor. However, the use of only a single rib would be inadequate to support the entire anterior column. If a rib graft is used, two or three measured struts of ribs should be tied together trans-osseously to reconstruct the full width of the body. A mesh cage filled with cortico-cancellous bone may also be used. In addition, 'onlay' grafting can be performed by inserting part of the excised rib between the vertebral body and anterior longitudinal ligament to increase the strength of the final union.

Segment salvage is associated with advantages, such as fusing fewer motion segments, reducing operative time, decreasing perioperative bleeding. However, short segment fixation may lead to implant failure and development of postoperative kyphosis.

However, our study had certain limitations. Our study is a retrospective study with a relatively small sample size. We had six patients in the pediatric age group only. In general, the treatment of spinal TB in the pediatric group varies from medical management to extensive debridement and an instrumented three-column reconstruction, depending on the presence of spinal instability (spine at risk signs), cord compression, and neurological deficit. Even in patients requiring surgery, the extent of surgical intervention could be quite variable depending the nature of the pathology and surgeon's preference. Hence, the protocols of surgical management of TB described in this study need to be applied carefully in the pediatric age group with proper planning on a case-to-case basis.

In our series, we found a combination of plain X-ray and MRI in three dimensions suffices the need for surgical planning with a good functional outcome. This saves the patient of an additional imaging modality (CT scan), which is of importance in a resource-poor setting, such as in India, where the study was conducted and also prevents additional radiation exposure. However, although MRI is a very informative study in terms of diagnosis of the pathology, it has limitations because it over-reads the extent of involvement. CT scan with three-dimensional reconstruction is often the study of choice for surgical planning and understanding the actual bone involvement and osseous anatomy. 
To summarize the limitations, a study with a larger sample size, with extra emphasis on pediatric population and inclusion of CT scan in the decision-making algorithm will validate our findings further.

\section{Conclusions}

To conclude, this method of deciding the level of fixation and segment salvage based on the coronal MRI provided good result in 64 of 65 patients, except for one patient in the segment sacrifice group who had graft buckling and resultant kyphosis. For segment salvage, having a clear three-dimensional idea regarding the viable remnant bone is important. Viable salvaged segment reduces the morbidity of the procedure, length of the construct, and unnecessary debridement without compromising neural recovery and fusion rate. Hence, additional cone down coronal cuts must be required when MRI is suggestive for spinal TB because it will help in the decision making.

\section{Conflict of Interest}

No potential conflict of interest relevant to this article was reported.

\section{Author Contributions}

Study design: Sudhir Srivastava, Sunil Bhosale; concept: Sudhir Srivastava; data collection: Nandan Marathe; review of literature: Nandan Marathe, Sunil Bhosale; statistics: Prajakta Bhide, Balgovind Raja; data analysis: Prajakta Bhide, Balgovind Raja, Shaligram Purohit; and editing and proof reading: Chetan Shende

\section{References}

1. Sai Kiran NA, Vaishya S, Kale SS, Sharma BS, Mahapatra AK. Surgical results in patients with tuberculosis of the spine and severe lower-extremity motor deficits: a retrospective study of 48 patients. J Neurosurg Spine 2007;6:320-6.

2. Turgut M. Spinal tuberculosis (Pott's disease): its clinical presentation, surgical management, and outcome: a survey study on 694 patients. Neurosurg Rev 2001;24:8-13.

3. Barnes PF, Bloch AB, Davidson PT, Snider DE Jr. Tuberculosis in patients with human immunodeficiency virus infection. N Engl J Med 1991;324:1644-50.

4. Moorthy S, Prabhu NK. Spectrum of MR imaging findings in spinal tuberculosis. AJR Am J Roentgenol 2002;179:979-83.

5. Rezai AR, Lee M, Cooper PR, Errico TJ, Koslow M. Modern management of spinal tuberculosis. Neurosurgery 1995;36:87-97.

6. Tuli SM. Results of treatment of spinal tuberculosis by "middle-path" regime. J Bone Joint Surg Br 1975;57:13-23.

7. Jain AK, Dhammi IK. Tuberculosis of the spine: a review. Clin Orthop Relat Res 2007;460:39-49.

8. Srivastava SK, Aggarwal RA, Bhosale SK, Roy K, Nemade PS. The versatile approach: a novel single incision combined with anterior and posterior approaches for decompression and instrumented fusion to treat tuberculosis of the thoracic spine. Asian Spine J 2017;11:294-304.

9. Alothman A, Memish ZA, Awada A, et al. Tuberculous spondylitis: analysis of 69 cases from Saudi Arabia. Spine (Phila Pa 1976) 2001;26:E565-70.

10. Jain AK. Tuberculosis of the spine: a fresh look at an old disease. J Bone Joint Surg Br 2010;92:905-13.

11. Bakhsh A. Medical management of spinal tuberculosis: an experience from Pakistan. Spine (Phila Pa 1976) 2010;35:E787-91.

12. Kirkman MA, Sridhar K. Posterior listhesis of a lumbar vertebra in spinal tuberculosis. Eur Spine J 2011;20:1-5.

13. Gupta SK, Mohindra S, Sharma BS, et al. Tuberculosis of the craniovertebral junction: is surgery necessary? Neurosurgery 2006;58:1144-50.

14. Topuz K, Kutlay AM, Simsek H, Colak A, Kaya S, Demircan MN. Effect of hyperbaric oxygen therapy on the duration of treatment of spinal tuberculosis. J Clin Neurosci 2009;16:1572-7.

15. Moon MS, Moon YW, Moon JL, Kim SS, Sun DH. Conservative treatment of tuberculosis of the lumbar and lumbosacral spine. Clin Orthop Relat Res 2002;(398):40-9.

16. Bhojraj S, Nene A. Lumbar and lumbosacral tuberculous spondylodiscitis in adults: redefining the indications for surgery. J Bone Joint Surg Br 2002;84:530-4.

17. Chadha M, Agarwal A, Singh AP. Craniovertebral tuberculosis: a retrospective review of 13 cases managed conservatively. Spine (Phila Pa 1976) 2007;32:1629-34.

18. Nene A, Bhojraj S. Results of nonsurgical treatment 
of thoracic spinal tuberculosis in adults. Spine J 2005;5:79-84.

19. Kotil K, Alan MS, Bilge T. Medical management of Pott disease in the thoracic and lumbar spine: a prospective clinical study. J Neurosurg Spine 2007;6:2228.
20. Parthasarathy R, Sriram K, Santha T, Prabhakar R, Somasundaram PR, Sivasubramanian S. Shortcourse chemotherapy for tuberculosis of the spine: a comparison between ambulant treatment and radical surgery: ten-year report. J Bone Joint Surg Br 1999;81:464-71. 